\title{
On the evolution of superposition of squeezed displaced number states with the multiphoton Jaynes-Cummings model
}

\author{
Faisal A. A. El-Orany ${ }^{1}$ and and A.-S. Obada ${ }^{2}$ \\ ${ }^{1}$ Department of Mathematics and computer Science, \\ Faculty of Science, Suez Canal University 41522, Ismailia, Egypt \\ 2 Department of Mathematics, Faculty of Natural Science, \\ Al-Azhar University, Nasr City 11884, Cairo, Egypt
}

(Dated: June 1, 2018)

\begin{abstract}
In this paper we discuss the quantum properties for superposition of squeezed displaced number states against multiphoton Jaynes-Cummings model (JCM). In particular, we investigate atomic inversion, photon-number distribution, purity, quadrature squeezing, Mandel $Q$ parameter and Wigner function. We show that the quadrature squeezing for three-photon absorption case can exhibit revivals and collapses typical to those occurring in the atomic inversion for one-photon absorption case. Also we prove that for odd number absorption parameter there is a connection between the evolution of the atomic inversion and the evolution of the Wigner function at the origin in phase space. Furthermore, we show that the nonclassical states whose the Wigner functions values at the origins are negative will be always nonclassical when they are evolving through the JCM with even absorption parameter.
\end{abstract} Also we demonstrate that various types of cat states can be generated via this system.

PACS numbers: $42.50 \mathrm{Dv}, 42.60 . \mathrm{Gd}$

\section{INTRODUCTION}

Jaynes-Cummings model (JCM) [1] is the simplest nontrivial example of two interacting quantum systems: a two-level atom and a single mode of the radiation field. In addition to its being exactly solvable, the physical system that it represents has become experimentally realizable with the Rydberg atom in high- $Q$ microwave cavities, e.g. [2]. Such experimental realization has stimulated an intense research devoted to highlighting and generalizing the original model. For example, this model has been extended to include, e.g., multiphoton [3, 4, 5], intensity-dependent coupling [6] and damping and dissipation [7]. Investigation of the JCM has produced many interesting effects, which have no classical analogue, such as revival-collapse phenomenon in the atomic inversion [8], quadrature squeezing [9] and sub-Poissonian statistics [10]. Furthermore, JCM has been used as a source for the nonclassical states by means of the conditional measurement technique [11]. For 
more details related to JCM reader can consult [12]. On the other hand, evolution of states with JCM is an important topic, which can give insight into the occurrence of the nonclassical effects and how can be controlled based on the values of the interaction parameters. In this respect we can mention, e.g., Shrödinger-cat states [13, 14, 15], displaced number states [16] and squeezed coherent states [17, 18, 19].

Recently, we have introduced a general class of quantum states as a single mode vibration of electromagnetic field suddenly squeezed-plus-displaced by a collection of two displacements $\phi$ out of phase with respect to each other [20, 21]. These states (SSNDS) are defined as

$$
|\alpha, r, n\rangle_{\epsilon}=\lambda_{\epsilon}[\hat{D}(\alpha)+\epsilon \hat{D}(-\alpha)] \hat{S}(r)|n\rangle
$$

where $\lambda_{\epsilon}$ is a normalization constant, $\hat{D}(\alpha)$ and $\hat{S}(r)$ are displacement and squeeze operator, respectively, while $\alpha$ and $r$ are displacement and squeeze parameters. $\epsilon=|\epsilon| \exp (i \phi)$, where $\phi$ is the relative phase and throughout the paper $\epsilon=0, i, 1,-1$ in correspondence with squeezed displaced number state, Yurke-Stoler-, even- and odd-squeezed-displaced number states. Also $|n\rangle$ denotes Fock state. Squeeze and displacement operators are given, respectively, by

$$
\hat{S}(r)=\exp \left[\frac{r}{2}\left(\hat{a}^{2}-\hat{a}^{\dagger 2}\right)\right], \quad \hat{D}(\alpha)=\exp \left(\hat{a}^{\dagger} \alpha-\hat{a} \alpha^{*}\right),
$$

where $\hat{a}$ and $\hat{a}^{\dagger}$ are annihilation and creation operators. The normalization constant $\lambda_{\epsilon}$ is given by

$$
\left|\lambda_{\epsilon}\right|^{-1}=1+|\epsilon|^{2}+2|\epsilon| \exp \left(-2|\beta|^{2}\right) \mathrm{L}_{n}\left(4|\beta|^{2}\right) \cos \phi
$$

with

$$
\beta=\alpha \cosh r+\alpha^{*} \sinh r
$$

and $L_{n}($.$) are the Laguerre polynomials. Furthermore, the density matrix \hat{\rho}_{f}(0)$ of this state can be written as

$$
\begin{aligned}
& \hat{\rho}_{f}(0)=\left|\psi_{f}^{(1)}(0)\right\rangle\left\langle\psi_{f}^{(1)}(0)\right| \\
& =\left|\lambda_{\epsilon}\right|^{2}\left(\hat{\rho}_{M}+\hat{\rho}_{I}\right)
\end{aligned}
$$

where $\left|\psi_{f}^{(1)}(0)\right\rangle=|\alpha, r, n\rangle_{\epsilon}$ and the subscript $f$ stands for the radiation field density matrix. The second line of (5) includes the statistical mixture part $\hat{\rho}_{M}$ and interference part $\hat{\rho}_{I}$ of the density matrix. The statistical mixture part has the form

$$
\hat{\rho}_{M}=\hat{D}(\alpha) \hat{S}(r)|n\rangle\left\langle\left. n\left|\hat{S}^{\dagger}(r) \hat{D}^{\dagger}(\alpha)+\right| \epsilon\right|^{2} \hat{D}(-\alpha) \hat{S}(r) \mid n\right\rangle\langle n| \hat{S}^{\dagger}(r) \hat{D}^{\dagger}(-\alpha)
$$


while the quantum interference part is

$$
\hat{\rho}_{I}=\epsilon^{*} \hat{D}(\alpha) \hat{S}(r)|n\rangle\left\langle n\left|\hat{S}^{\dagger}(r) \hat{D}^{\dagger}(-\alpha)+\epsilon \hat{D}(-\alpha) \hat{S}(r)\right| n\right\rangle\langle n| \hat{S}^{\dagger}(r) \hat{D}^{\dagger}(\alpha)
$$

This part, i.e. $\hat{\rho}_{I}$, of the density matrix includes information about the quantum interference between the components of the state. On the other hand, in the number state basis (1) takes the form [21, 22]

$$
|\alpha, r, n\rangle_{\epsilon}=\sum_{m=0}^{\infty} C_{m}(\alpha, r, n, \epsilon)|m\rangle,
$$

where the distribution coefficient has the form

$$
\begin{array}{r}
C_{m}(\alpha, r, n, \epsilon)=\frac{\lambda_{\epsilon}\left(\frac{\tanh r}{2}\right)^{\frac{m}{2}}}{\sqrt{n ! m ! \cosh r}}\left[1+(-1)^{(n+m)} \epsilon\right] \exp \left[\frac{\alpha^{2} e^{2 r}}{2}(\tanh r-1)\right] \\
\times \sum_{j=0}^{\min (m, n)} \frac{n ! m !}{j !(n-j) !(m-j) !}\left[\frac{2}{\sqrt{\sinh 2 r}}\right]^{j}\left[\frac{-\tanh r}{2}\right]^{\frac{(n-j)}{2}} \mathrm{H}_{n-j}\left(\frac{i \alpha}{\sqrt{\sinh (2 r)}}\right) \mathrm{H}_{m-j}\left(\frac{e^{r} \alpha}{\sqrt{\sinh (2 r)}}\right),
\end{array}
$$

whereas $\mathrm{H}_{m}($.$) is the Hermite polynomial of order m$ and $\alpha$ has been considered to be real. The photon-number distribution $P(m)$ of the state (1) is

$$
P(m)=\left|C_{m}(\alpha, r, n, \epsilon)\right|^{2}
$$

For completeness, it has been shown that states (11) can be generated by the so-called quantum state engineering and also by trapped ion [20, 21]. The quantum properties of these states reveal that they can exhibit sub-Poissonian statistics, quadrature squeezing and oscillations in photonnumber distribution. Their phase properties from the point of view of the Pegg-Barnett Hermitian phase formalism [22] show that there are two regimes controlling the behaviour based on whether the superposition is macroscopic $(\alpha>>1)$ or microscopic $(\alpha \leq 1)$. For such superposition the nonclassical effects are more pronounced in the microscopic regime. Moreover, the influence of thermal noise on the behaviour of (11) has been considered in [23] showing that the correlation between different oscillators is essentially responsible for the occurrence of the nonclassical effects, which is similar to that of Schrödinger-cat states with thermal noise [24].

For convenience we mention that a new set of squeezed states using group-theoretical methods has been presented earlier, e.g. [25]. Actually, the definition of this set is based on the HolsteinPrimakoff realization of both $S U(2)$ and $S U(1,1)$. It has been shown that such type of states can exhibit interesting squeezing properties, depending in a characteristic way on the dimension of the irreducible unitary representation adopted. Moreover, the multiphoton, many-mode squeezed 
states for $S U(n)$, using a generalization Holstein-Primakoff realization have been also reported [26]. Finally, a long list of references related to different types of squeezed states can be found in the recent review [27].

In this paper we study the evolution of the states (1) with the multiphoton Jaynes-Cummings model. The motivation behind this work is to determine the evolution of the nonlinear process of the multiphoton transition in the presence of quantum interference of two squeezed displaced number states. Also, as we will show below, there are some intriguing features such as information about atomic inversion of the JCM can be obtained from quadrature squeezing of the nonlinear $\mathrm{JCM}$, and also there is a connection between the evolution of the Wigner function at the origin in phase space and the atomic inversion. In addition to that this work gives a generalization to several results given in the literatures earlier, e.g. [13]-[19]. Actually, there is some merit in having the most general results.

The Hamiltonian controlling the system in the rotating wave approximation (RWA) is

$$
\frac{\hat{H}}{\hbar}=\omega_{0} \hat{a}^{\dagger} \hat{a}+\omega_{a} \hat{\sigma}_{z}+g\left(\hat{a}^{k} \hat{\sigma}_{+}+\hat{a}^{\dagger k} \hat{\sigma}_{-}\right)
$$

where $\hat{\sigma}_{ \pm}$and $\hat{\sigma}_{z}$ are the Pauli spin operators; $\omega_{0}$ and $\omega_{a}$ are the frequencies of cavity mode and the atomic transitions, respectively; $g$ is the atom-field coupling constant and $k$ is the absorption parameter.

Throughout the paper we assume that the atom is initially prepared in its excited state $|+\rangle$ and the field is in the SSDNS (11). In this case the initial density matrix for the system is

$$
\hat{\rho}(0)=|+\rangle\left\langle+|\bigotimes| \psi_{f}^{(1)}(0)\right\rangle\left\langle\psi_{f}^{(1)}(0)\right|,
$$

where $\otimes$ stands for the direct product. Working in the interaction picture and considering the resonance case $\omega_{0}=k \omega_{a}$, after well-known analytical procedures, the time evolution density matrix describing the system is

$$
\begin{aligned}
& \hat{\rho}(T)=|\psi(T)\rangle \otimes\langle\psi(T)| \\
& =\sum_{m, m^{\prime}=0}^{\infty} C_{m}(\alpha, r, n, \epsilon) C_{m^{\prime}}^{*}(\alpha, r, n, \epsilon)\{\cos [T \sqrt{h(m, k)}]|m,+\rangle \\
& -i \sin [T \sqrt{h(m, k)}]|m+k,-\rangle\} \otimes\left\{\left\langle m^{\prime},+\right| \cos \left[T \sqrt{h\left(m^{\prime}, k\right)}\right]+i\left\langle m^{\prime}+k,-\right| \sin \left[T \sqrt{h\left(m^{\prime}, k\right)}\right]\right\},
\end{aligned}
$$

where $T=g t$ is the scaled time, $|-\rangle$ is atomic ground state and $h(m, k)=\frac{(m+k) !}{m !}$. Also $|\psi(T)\rangle$ is the dynamical state vector of the system. From (13) the entanglement of the two interacting quantum 
systems is readily apparent. To evaluate quantities associated with atom (or field) we have to trace (13) over the field (or the atom) system. As it is obvious the system includes various parameters, which make such analysis difficult. For this reason throughout the paper we restrict ourselves to the parameters, which can give significant results. Also throughout the text the statement standard JCM means $k=1$, the optical cavity field and atom are initially prepared in coherent states and in the atomic excited state, respectively.

The paper is organized as follows: In section 2 atomic inversion is investigated. Section 3 is devoted to the photon-number distribution and purity. In section 4 Mandel $Q$ parameter and quadrature squeezing are discussed. In section 5 Wigner function is demonstrated. Conclusions are summarized in section 6 .

\section{ATOMIC INVERSION}

In this section we discuss the revival-collapse phenomenon in the evolution of the atomic inversion for the density matrix (13). This phenomenon is a pure quantum mechanical effect and having its origin in the granular structure of the photon-number distribution of the initial field [28]. Furthermore, this phenomenon has been realized experimentally in the sense that the state of the atomic beam leaving the cavity is monitored by ionization detectors [2]. On the other hand, the revival-collapse phenomenon has been seen also in nonlinear optics for the single-mode mean-photon number of the Kerr nonlinear coupler [29] when the modes are initially prepared in coherent light, however, in this case the origin of the occurrence of such phenomenon is in the presence of nonlinearity in the system (third-order nonlinearity specified by the cubic susceptibility). We should stress here that the analytical formulae of both the mean-photon number of the Kerr coupler and of the atomic inversion of the JCM (under the harmonic approximation) [8] are similar. This remark makes the occurrence of the collapse-revival pattern in the Kerr coupler is an expected result.

Now the atomic inversion for the density matrix (13) reads

$$
\begin{aligned}
& \left\langle\hat{\sigma}_{z}(T)\right\rangle=\operatorname{Tr}_{f}\left[\hat{\sigma}_{z}(0) \hat{\rho}(T)\right] \\
& =\sum_{m=0}^{\infty} P(m) \cos [2 T \sqrt{h(m, k)}]
\end{aligned}
$$

where $P(m)$ is given by (10) and the subscript $f$ on the right hand side means that we trace over the field system. Firstly, it is worth reminding that the behaviour of the $\left\langle\hat{\sigma}_{z}(T)\right\rangle$ of squeezed 

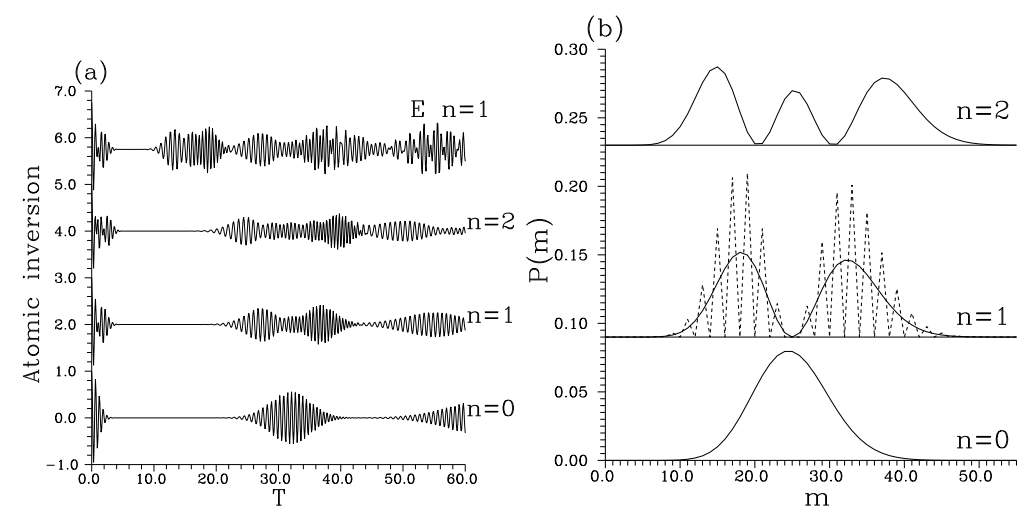

FIG. 1: The atomic inversion (a) and the corresponding initial photon-number distribution (b) for the Yurke-Stoler displaced number states and for $\alpha=5, k=1$; the different values of $n$ are specified in the figures. In Fig. 1a $E$ denotes that this curve is associated with the even displaced number states whose $P(m)$ is given in Fig. $1 \mathrm{~b}$ as dashed curve. Also $\left\langle\hat{\sigma}_{z}(T)\right\rangle$ and $P(m)$ are shifted from bottom to top by $0,2,4,6$ and $0,0.09,0.23$, respectively.

coherent light exhibits various interesting effects [17, 18]. For instance, it has been shown that for strong coherent contribution [17] the collapse time depends on the direction of the squeezing and for certain values of the squeezing parameter the response of the atom is similar to that of the chaotic radiation field. On the contrary, for strong squeezing contribution [18] the dynamical response of the atomic inversion shows echoes after each revival resulting from the interference effect. We start our discussion for $k=1$ and the cavity field is in superposition of displaced number states (see Figs. 1 for given values of the parameters). Actually, the superposition of displaced number states can exhibit strong sub-Poissonian statistics as well as quadrature squeezing. Also they can be generated via the driven Jaynes-Cummings model or via trapped ion [30, 31]. We proceed, it has been shown that there is a close correspondence between the number of peaks (with Poissonian envelopes) involving in $P(m)$ and the number of occurred revivals in $\left\langle\hat{\sigma}_{z}(T)\right\rangle[16]$. In other words, the envelope of each revival is a readout of the photon distribution, in particular, for slowly varying photon distribution [32] provided that the atom is in the excited or in the ground state. Actually, for appropriate values of the parameters and when $\epsilon=i$, the connection between the peaks in $P(m)$ and the revivals in $\left\langle\hat{\sigma}_{z}(T)\right\rangle$ can be explained as follows [18]: The different peaks in $P(m)$ have slightly different local mean photon numbers $\bar{m}_{j}$ (see Fig. 1b) and thus each peak of $P(m)$ gives its own revival-collapse pattern, which interfere with that of the others to produce a very complicated structure. In this case we have a sequence of the revivals with slightly different periods $T_{j}=\pi \sqrt{m_{j}}, \quad j=0,1, \ldots$. The echoes in the first revival will be separated by the intervals $\left(T_{j+1}-T_{j}\right)$ whereas those in the second revival will be separated by $2\left(T_{j+1}-T_{j}\right)$ 


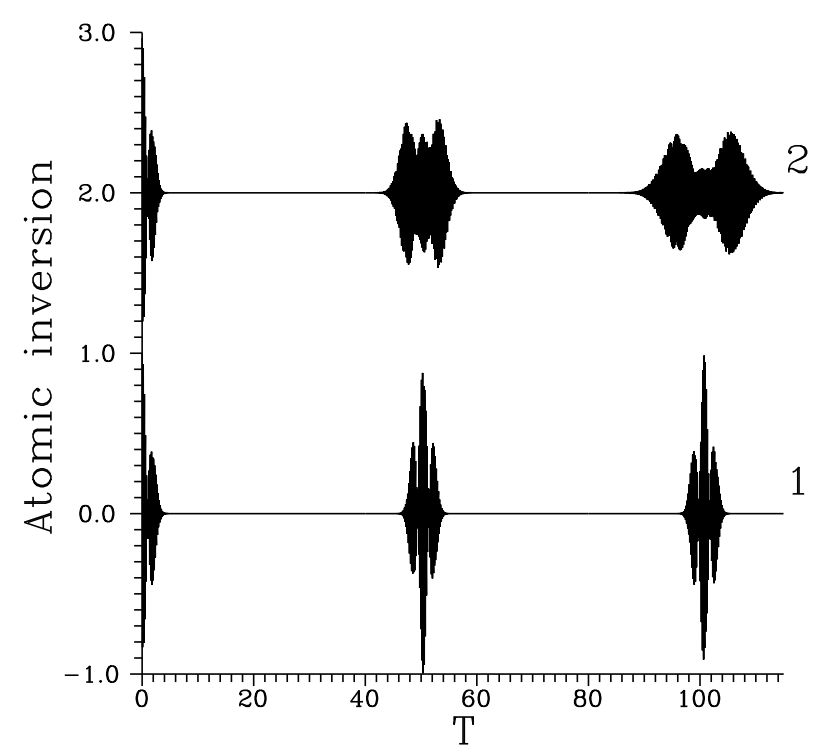

FIG. 2: The atomic inversion $\left\langle\hat{\sigma}_{z}(T)\right\rangle$ for the asymptotic (curve 1) and the exact $2+\left\langle\hat{\sigma}_{z}(T)\right\rangle$ (curve 2) cases for even displaced number state with $\alpha=16, k=1, r=0$ and $n=1$.

and so on. Such a systematic behaviour depends upon the chosen values of the parameters, in particular, $n$ (compare different curves in Fig. 1a). Furthermore, from this figure one can observe that the ringing revivals are remarkable for the case $n=2$. For large values of $n$ we noted that the chaotic behaviour is dominant and the collapse time is shortened. Now we turn our attention to the superimposed case, i.e. the even or odd case (see curve E in Fig. 1a). From this curve it is clear that the collapse time is smaller than that of the statistical mixture case owing to the interference in phase space. Precise information about this situation can be obtained from the asymptotic form for the atomic inversion, which can be evaluated via the harmonic approximation technique [18]. For this purpose and for the sake of simplicity we confine ourselves to $|\alpha|>>1, k=1, n=1$ and $r=0$. In this case the argument of $\cos ($.$) in (14) can be expressed as [18]:$

$$
\sqrt{n+1}=\sqrt{\langle\hat{n}\rangle+n+1-\langle\hat{n}\rangle} \simeq \frac{1}{2}\left(\eta_{1}+\eta_{2} n\right)
$$

where

$$
\eta_{1}=\sqrt{\langle\hat{n}\rangle}+\frac{1}{\sqrt{\langle\hat{n}\rangle}} \quad \eta_{2}=\frac{1}{\sqrt{\langle\hat{n}\rangle}}
$$

and $\langle\hat{n}\rangle$ is the initial mean-photon number for the state under consideration. On substituting (15) into (14) and after some minor algebra we arrive at 


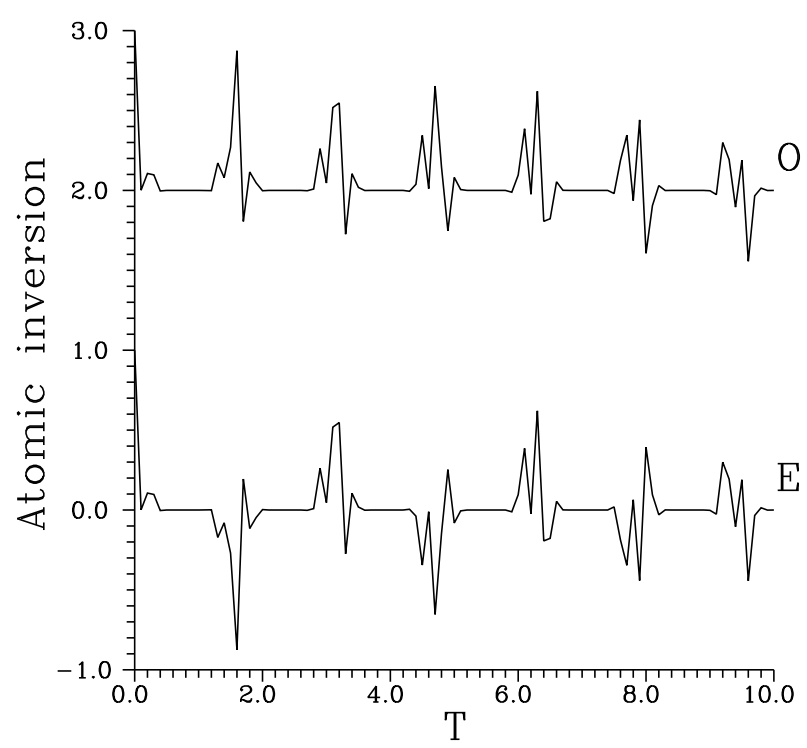

FIG. 3: The atomic inversion for even displaced Fock state (E-curve- $\left.\left\langle\hat{\sigma}_{z}(T)\right\rangle\right)$ and odd displaced Fock state (O-curve- $\left.2+\left\langle\hat{\sigma}_{z}(T)\right\rangle\right)$ for $\alpha=5, \quad k=2, \quad n=1$ and $r=0$.

$$
\begin{aligned}
& \left\langle\hat{\sigma}_{z}(T)\right\rangle=\lambda_{\epsilon}\left\{\left(1+|\epsilon|^{2}\right) f_{1}(T) \cos \left[T\left(\eta_{1}+\eta_{2}\right)+|\alpha|^{2} \sin \left(\eta_{2} T\right)\right]\right. \\
& \left.+2|\epsilon| f_{2}(T) \cos \phi \cos \left[T\left(\eta_{1}+\eta_{2}\right)-|\alpha|^{2} \sin \left(\eta_{2} T\right)\right]\right\}
\end{aligned}
$$

where

$$
\begin{aligned}
& f_{1}(T)=\left[1-4|\alpha|^{2} \sin ^{2}\left(\frac{\eta_{2} T}{2}\right)\right] \exp \left[-2|\alpha|^{2} \sin ^{2}\left(\frac{\eta_{2} T}{2}\right)\right] \\
& f_{2}(T)=\left[1-4|\alpha|^{2} \cos ^{2}\left(\frac{\eta_{2} T}{2}\right)\right] \exp \left[-2|\alpha|^{2} \cos ^{2}\left(\frac{\eta_{2} T}{2}\right)\right] .
\end{aligned}
$$

In (17), $f_{1}(T)$ and $f_{2}(T)$ are the envelope functions associated with the statistical-mixture part (6) and the interference part (7) of the optical cavity field density matrix, respectively. These functions are periodic with period $2 \pi \sqrt{\langle\hat{n}\rangle}$ and their maxima occur for $T_{R}^{(S)}=2 \pi \sqrt{\langle\hat{n}\rangle}$ and $T_{R}^{(I)}=\pi \sqrt{\langle\hat{n}\rangle}$ where the superscripts $(S)$ and $(I)$ mean that the times are associated with the statistical-mixture and interference parts, respectively, and the subscript $R$ denotes the revival time. It is obvious that $T_{R}^{(I)}=\frac{1}{2} T_{R}^{(S)}$, which is typical with that of the Schrödinger-cat states case [13]. Further, the term 1 in the square bracket of (18) corresponds to the coherent component in the optical cavity, whereas the second term is coming from the Fock state $|1\rangle$. In other words, the main revival associated with the initial coherent light is splitted into three parts showing subsidiary revivals (echoes) in the atomic inversion, e.g. for the statistical-mixture part splitting occurs at $\sin \left(\frac{T}{2 \sqrt{\langle\hat{n}\rangle}}\right)= \pm \frac{1}{2|\alpha|}$. 

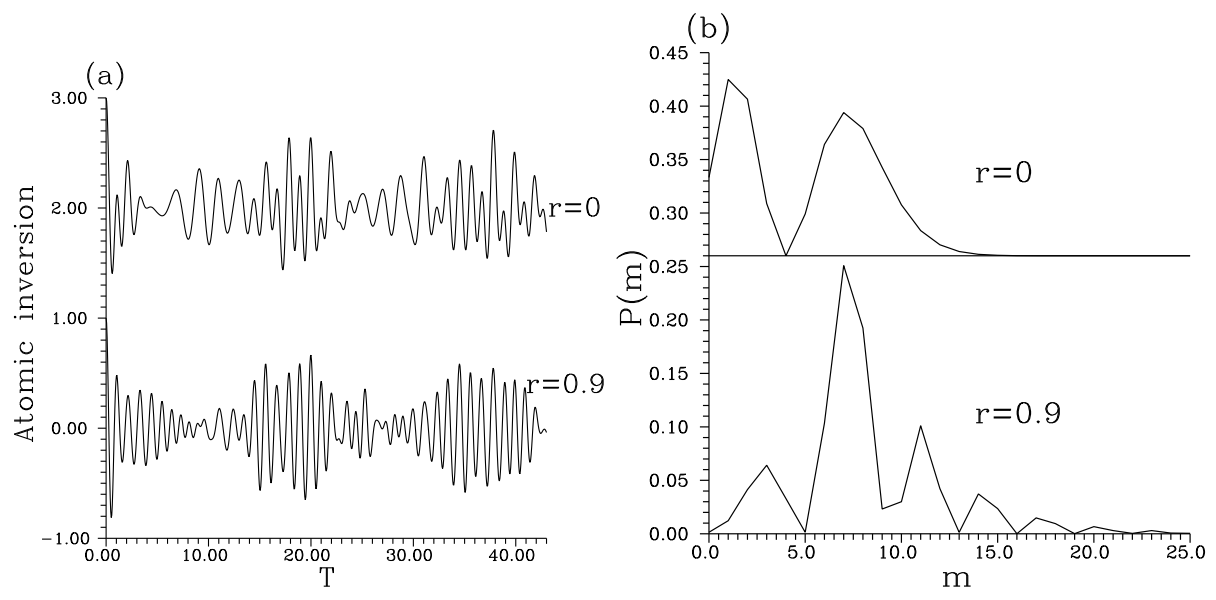

FIG. 4: The atomic inversion (a) and the corresponding initial photon-number distribution (b) for $\alpha=$ $3, \epsilon=i, \quad k=1, \quad n=1$ and different values of $r$ as indicated. $\left\langle\hat{\sigma}_{z}(T)\right\rangle$ and $P(m)$ are shifted from bottom to top by 0,2 and $0,0.26$, correspondingly.

Furthermore, we have checked the case $n=2$ and found that, e.g., the statistical-mixture-part envelope splits into four parts controlled by the equation

$$
8|\alpha|^{4} \sin ^{4}\left(\frac{\eta_{2} T}{2}\right)-8|\alpha|^{2} \sin ^{2}\left(\frac{\eta_{2} T}{2}\right)+1=0 .
$$

It is evident that the Fock state $|2\rangle$ develops two additional terms in the asymptotic form (19), which provide more echoes in $\left\langle\hat{\sigma}_{z}(T)\right\rangle$ compared to that of the case $|1\rangle$. In Fig. 2 we compare the behaviour of the asymptotic formula (17) with that of the exact one (14) for the given values of the parameters. This figure reveals agreement and disagreement between the exact and asymptotic behaviour. Qualitatively, they are approximately in agreement in the locations of the revivalcollapse pattern as well as each revival includes three portions, but the corresponding revivals cannot provide typical amplitude and shape. For the exact case (curve 2) the revivals are broader than those for the asymptotic case. This problem can be overcome by means of the higher-order approximation in (15) [18], however, the price that should be paid, is that we cannot obtain a simple closed form for $\left\langle\hat{\sigma}_{z}(T)\right\rangle$. On the other hand, excluding the initial revival in Fig. 2, the origins of the first and second revivals, respectively, are statistical-mixture and interference parts of the initial density matrix of the radiation field. In conclusion the interference in phase space decreases the collapse time and the excitation number $n$ yields echoes.

The issue we would like to discuss here is that, qualitatively, the nature of quantum interference in phase space cannot manifest itself in the behaviour of the atomic inversion for the one-photon JCM. This is not the case for the multiphoton JCM where, e.g., the difference between the evenstate and odd-state cases becomes clear, in particular, for $k=2$. Actually, the two-photon JCM 
has been extensively studied and extended in many direction (e.g. [33]). Information about the case $k=2$ is shown in Fig. 3 for given values of the interaction parameters. Clearly, the revivals in the two-photon case are much more compact than those in the one-photon case. Physically speaking in the two-photon process the phase correlation of the two superimposed optical cavities is transferred to the atomic dynamics [15]. This is in contrast with the one-photon case where the atom absorbs only one photon at a time. Furthermore, such behaviour can be realized via the asymptotic form of this case, where we use the asymptotic expansion [15]

$$
\sqrt{(n+1)(n+2)} \simeq \frac{3}{2}+n
$$

Comparison between (15) and (20) leads to the fact that the asymptotic results related to the two-photon case can be obtained from those of the one-photon case by simply setting $\eta_{1}=3$ and $\eta_{2}=2$. In this case the envelope functions revive periodically with period $T=\pi$ and then the revival time is $T=\pi / 2$. So that the collapse and revival times become approximately intensity independent and this is responsible for such a systematic behaviour in the evolution of the atomic inversion. On the other hand, we have noted that for $k \geq 3$ the revivals interfere to produce chaotic population after the first collapse [5], i.e. the collapse-revival phenomenon of the atomic inversion for multiphoton Jaynes-Cummings model is more dramatic.

We conclude this section by demonstrating that including squeezing in the superimposed optical cavity can stimulate revival-collapse pattern in the behaviour of $\left\langle\hat{\sigma}_{z}(T)\right\rangle$. In doing so, we plot Figs. 4 for the shown values of the parameters. From Fig. $4 \mathrm{~b}$ one can observe that for $r=0, P(m)$ exhibits two peaks, which are smooth Gaussian peaks. Such structure leads to chaotic behaviour in $\left\langle\hat{\sigma}_{z}(T)\right\rangle$ (see Fig. 4a for the corresponding case) and can be explained as follows: the second peak in $P(m)$ gives the familiar collapse-revival pattern in $\left\langle\hat{\sigma}_{z}(T)\right\rangle$, whereas the first peak (which is incomplete one) provides oscillatory behaviour. Therefore, the competition between these two processes produces such behaviour. On the other hand, when $r \neq 0$, i.e. squeezing in the optical cavity starts to play a role, one can observe that $P(m)$ has oscillatory behaviour with smooth Gaussian envelope, which causes the well-known revival-collapse phenomenon in $\left\langle\hat{\sigma}_{z}(T)\right\rangle$. This situation is similar to that of squeezed number states [16].

\section{PHOTON-NUMBER DISTRIBUTION AND PURITY}

In this section we discuss the evolution of the photon-number distribution $P(m, T)$ and purity $T_{f}$ for the system under consideration. As is well known photon-number distribution can be measured 

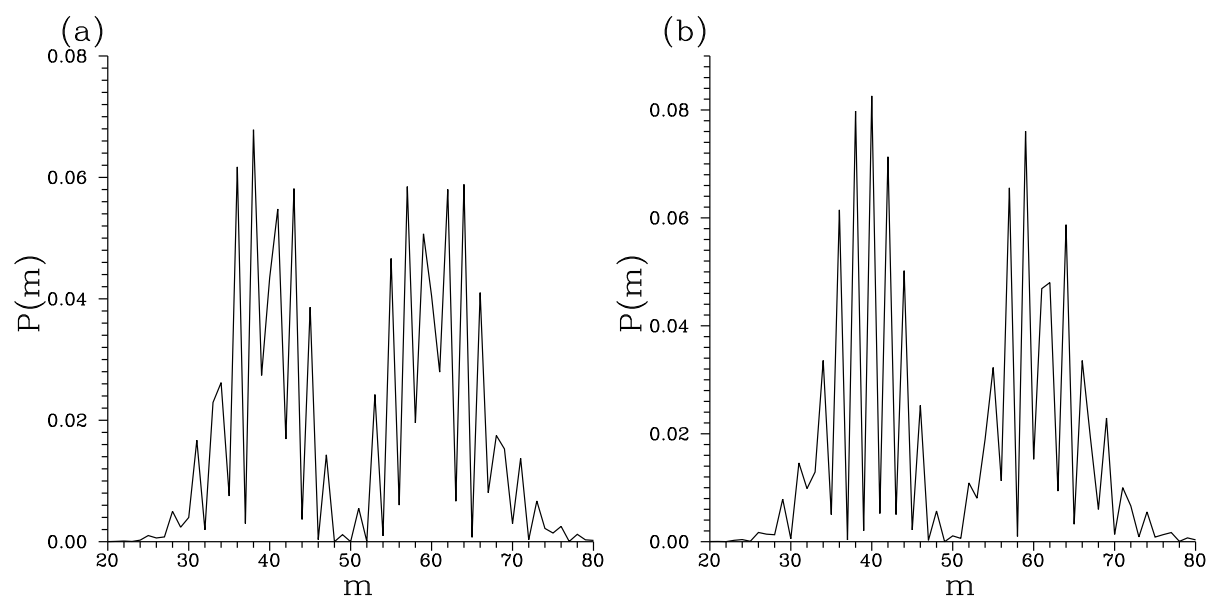

FIG. 5: The photon-number distribution for $\epsilon=0, \alpha=7, k=1$ and $n=1$ and for (a) $T=22.21$; (b) $T=20.64$.

by photon detectors based on the photoelectric effect. Also, the characters of the field can be emphasized via $P(m, T)$, which only at particular values of the interaction time resemble those of the initial pure states. On the other hand, investigation of the purity for the present quantum dynamical system is important to know how far the atom and the field are entangled.

We start the discussion by analyzing $P(m, T)$, which has the form

$$
P(m, T)=P(m) \cos ^{2}[T \sqrt{h(m, k)}]+P(m-k) \sin ^{2}[T \sqrt{h(m-k, k)}],
$$

where $P(m)$ are given by (10). As the optical cavity field starts to interact with the atom the initial field distribution changes because of the quantum dynamics described by the Hamiltonian (11), however, at specific times the field and the atom subsystems return to their initial states. Basically such behaviour depends upon the values of both the interaction time and the absorption parameter. To be more specific, this behaviour is established well for $k=2$ and 4 , as we will show shortly. On the other hand, for the standard JCM at one-half of the revival time pure-superposition states can be generated and the optical cavity field and the atom become completely disentangled [34, 35, 36, 37]. These states are well established in the strong-intensity regime, i.e. large values of $|\alpha|$. Now we discuss the possible occurrence of such behaviour in the present system. To achieve our goal in a convenient way we restrict ourselves to the case $\epsilon=0$ and $r=0$. In figures 5 we plot $P(m, T)$ for $T=\pi \sqrt{\langle\hat{n}(0)\rangle}=22.21$ (one-half of the revival time) (a) and $\pi \sqrt{\langle\hat{n}(0)\rangle}-0.5 \pi=20.64$ (close to one-half of the revival time) (b) and for the given values of the parameters. Actually, at these times the optical cavity field will be approximately in pure states this will be noticeable in the behaviour of the purity (see Fig. 7 below). Furthermore, Figs. 5 reveal that the optical cavity collapses to superposition of displaced number states, particularly, superposition of $\hat{D}(\alpha)|1\rangle$. This 
is obvious where $P(m, T)$ has oscillatory behaviour with two-Gaussian envelopes representative to such type of states [30]. Of course the oscillations in $P(m, T)$ are resulting from the quantum interference in phase space. The quantum properties for such type of states (,i.e., superposition of displaced number states) have been discussed in [30] and they have been generated also in a state-selective measurement [31]. We proceed, comparison of Fig. 5a and Fig. 5b reveals that the oscillations in $P(m, T)$ are extremely sensitive to the values of the interaction time. Furthermore, comparison between the oscillatory behaviour in the two peaks in Fig. 5a (or in Fig. 5b) shows that a clear asymmetry and this is connecting with the asymmetry in the initial photon-number distribution of the states $\hat{D}(\alpha)|1\rangle$ (see Fig. 1b). Actually, by changing the types of the initial state we can obtain different forms of cat states.

Now we discuss the case $k=2$ (two-photon absorption) whose scaled revival time is $T_{R}=\pi$, where we still consider $\epsilon=0$. We found that the optical cavity field at $T=s T_{R}$ where $s$ is positive integer, collapses to pure state typical (or similar to) the initial states, while the atom becomes either in the ground state or in the excited state. In other words, the atom and the field subsystems become completely disentangled at these specified times. Such behaviour can be analytically determined provided that $\alpha$ is large and $n$ is finite through the substitution of both $T=T_{R}=\pi$ and (20) into the state vector obtained from (13), we arrive at:

$$
\begin{aligned}
& \left|\psi\left(T=T_{R}\right)\right\rangle=\sum_{m=0}^{\infty} C_{m}(\alpha, r, n)\left\{\cos \left[T_{R}\left(m+\frac{3}{2}\right)\right]|m,+\rangle-i \sin \left[T_{R}\left(m+\frac{3}{2}\right)\right]|m+2,-\rangle\right\} \\
& =\left|\psi_{f}^{(2)}(0)\right\rangle \otimes \exp \left(\frac{i}{2} \pi\right)|-\rangle,
\end{aligned}
$$

where $\left|\psi_{f}^{(2)}(0)\right\rangle$ denotes the field state having the form

$$
\left|\psi_{f}^{(2)}(0)\right\rangle=\sum_{m=0}^{\infty} C_{m}(\alpha, r, n) \exp (i m \pi)|m+2\rangle .
$$

Expression (23) shows that the cavity field possesses photon distribution as that of the initial one, but with two-photon shift. On the other hand, by applying the above procedures for the case $T=2 T_{R}=2 \pi$, one can easily show that the system returns back to its initial form, i.e. the atom is in its excited state and the field is in $\left|\psi_{f}^{(1)}(0)\right\rangle$ given by (1) with $\epsilon=0$. Generally, we conclude that the system evolves periodically according to the following relation:

$$
\left|\psi_{f}^{(1)}(T)\right\rangle= \begin{cases}\left|\psi_{f}^{(2)}(0)\right\rangle & \text { for } T=s T_{R} \text { and } s=1,3,5, . . \\ \left|\psi_{f}^{(1)}(0)\right\rangle & \text { for } T=s T_{R} \text { and } s=2,4,6, . .,\end{cases}
$$




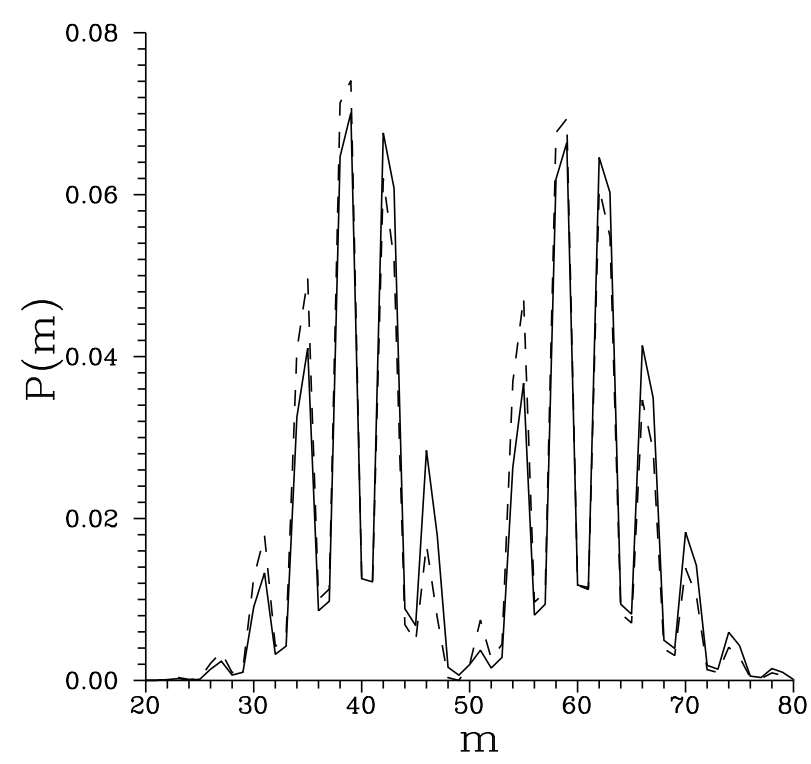

FIG. 6: The photon-number distribution for $k=2, T=\frac{1}{4} \pi$ and the other parameters as those in Fig. 5.

Expression (24) can be understood as follows: when the interaction is going on the atom decays (spontaneously and stimulately) from the excited state to the ground state contributing its photon to the cavity field and the system becomes disentangled during interaction time $T=T_{R}$. This means that the atomic excitation energy is transferred to the field [36], i.e. an information about the initial state of the atom is stored in the field. Furthermore, when the interaction continues the feed back between field and atom occurs and after $T=2 T_{R}$ the system returns back to its initial form. This behaviour is also visible in the evolution of the atomic inversion and reflects the periodic nature of the system. We should stress that the origin of such behaviour is two-fold: (i) We have considered the system is completely isolated (the interaction with the environment is neglected).

(ii) We treat the system in the RWA, which ensures that whenever $k$ photons are lost from the field the atomic state must change from ground to excited states, or vice versa. Furthermore, there is a similarity between the present behaviour and that of the frequency conversion device in which the energy interchanges periodically between the signal and idler modes. On the other hand, it has been shown that for the case $k=2$ and at the quarter of the revival time superposition states can be generated [36]. Presently we show analytically using a similar technique as that in [34] that similar behaviour can occur. We restrict ourselves to the case $\epsilon=0, n=1$ and $\alpha$ is very large. On substituting $T=\frac{1}{4} T_{R}=\frac{1}{4} \pi$ and (20) into the state vector (13), we obtain 

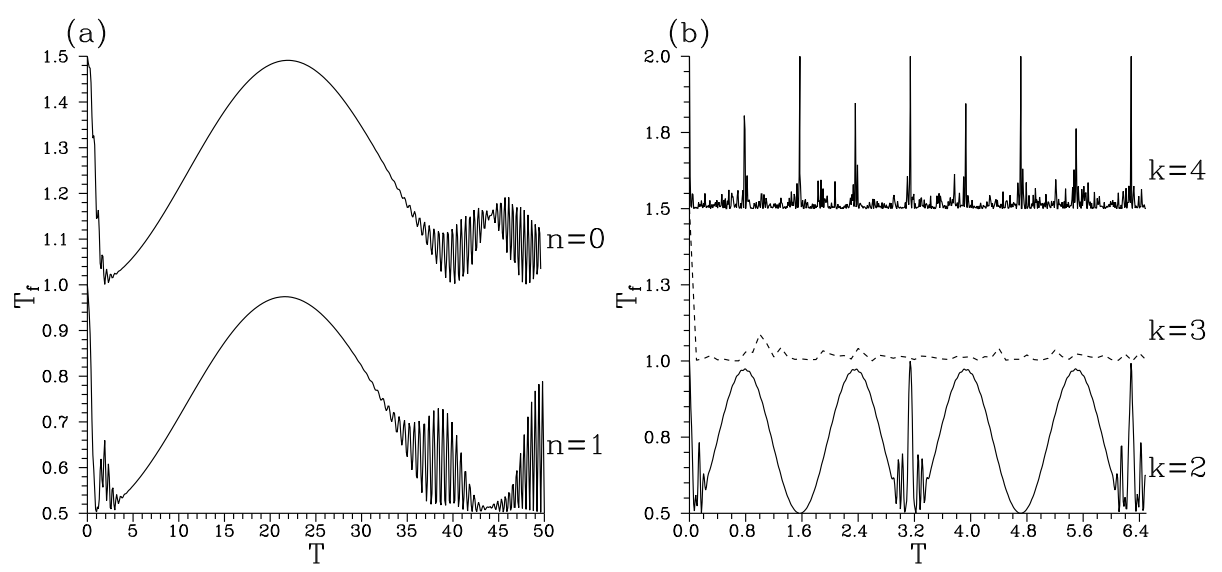

FIG. 7: The purity against the scaled time $T$ for: a) $(\alpha, k, \epsilon)=(7,1,0)$; and b) $(\alpha, n, \epsilon)=(7,1,0)$. In (a) for $n=0$ we plot $T_{f}+0.5$. In (b) for $k=3$ and 4 we plot $T_{f}+0.5$ and $T_{f}+1$, respectively.

$$
\begin{aligned}
& \left|\psi\left(T=\frac{1}{4} T_{R}\right)\right\rangle=\sum_{m=0}^{\infty} C_{m}(\alpha, r, n)\left\{\cos \left[\frac{1}{4} T_{R}\left(m+\frac{3}{2}\right)\right]|m,+\rangle-i \sin \left[\frac{1}{4} T_{R}\left(m+\frac{3}{2}\right)\right]|m+2,-\rangle\right\} \\
& =\sum_{m=0}^{\infty} \cos \left[\frac{1}{4} T_{R}\left(m+\frac{3}{2}\right)\right]\left\{C_{m}(\alpha, r, n)|+\rangle+i C_{m-2}(\alpha, r, n)|-\rangle\right\}|m\rangle .
\end{aligned}
$$

For the case $n=1$ the relation between $C_{m}(\alpha, r, n)$ and $C_{m-2}(\alpha, r, n)$ reads

$$
C_{m-2}(\alpha, r, n)=\sqrt{\frac{m^{2}}{\alpha^{4}}-\frac{m}{\alpha^{4}}}\left[\frac{m-\alpha^{2}-2}{m-\alpha^{2}}\right] C_{m}(\alpha, r, n) .
$$

It is worth remembering that the initial photon distribution has two-peak structure (see Fig. 1b) each of which has a Poissonian envelope. Further, for very large values of $\alpha$ one can find out that the terms contribute effectively to the summation in (25) are those for which the values of $\alpha^{2}$ and $m$ are comparable. Therefore, the first term in the square root of (26) tends to unity while the second one tends to zero. Also the terms in the square bracket reduces to unity where $\alpha^{2}>>2$. Considering all these suggestions, (25) can be rewritten as

$$
\left|\psi\left(T=\frac{1}{4} T_{R}\right)\right\rangle=\sum_{m}^{\infty} C_{m}(\alpha, r, n) \cos \left[\frac{1}{4} T_{R}\left(m+\frac{3}{2}\right)\right]|m\rangle \bigotimes\{|+\rangle+i|-\rangle\} .
$$

From this expression it is clear that at the quarter of the revival time the atom and the field become asymptotically disentangled and the atom evolves in an atomic superposition state, whereas the cavity field collapses to superposition of states whose forms are somewhat similar to those given in 38]. From (27) it is clear that $\cos \left[\frac{1}{4} T_{R}\left(m+\frac{3}{2}\right)\right] \neq 0$ for $m$ integer meaning that the $P(m, T)$ has oscillatory behaviour but cannot have perfect oscillations (,i.e., $P\left(m, T=T_{R} / 4\right)=0$ ) more than 
those included in the initial distribution of the optical cavity. This behaviour is clear in Fig. 6 where we plot the $P(m, T)$ at $T=T_{R} / 4$ for the same situation as in Fig. 5 but $k=2$. Furthermore, in Fig. 6 the solid and dashed curves are associated with the exact (21) and asymptotic (27) forms of $P(m, T)$. From Fig. 6 one can observe that the solid and dashed curves include significantly similar behaviour. In other words, under these conditions the asymptotic expression (27) can be used to describe the optical cavity field well. It is worth mentioning that approximate perfect oscillations can be achieved in the behaviour of this case if one uses the replacement $T_{R} \rightarrow T_{R} \pm 0.08$, however, the behaviour of $P(m, T)$ will be very close to that in Fig. 5b. Furthermore, in (11) if $\hat{a}^{k}$ replaced by $\hat{a} \sqrt{\hat{a}^{\dagger} \hat{a}}$ (i.e., the system becomes intensity-dependent JCM) the system can generate perfectly even and odd superposition of displaced number states (considering the initial state is the displaced Fock state) according to the atom is prepared initially in the ground and in the excited states, respectively.

We close this section by analyzing the purity for the optical cavity field via the relation $T_{f}=$ $\operatorname{Tr}\left[\hat{\rho}_{f}^{2}(T)\right]$ where $\hat{\rho}_{f}(T)=\operatorname{Tr}_{a}[\hat{\rho}(T)]$ is the field reduced density matrix, whereas $\hat{\rho}(T)$ is the total density matrix of the system (13). When $T_{f}=1$ the reduced density matrix describes pure state, however, for $T_{f}<1$ the field will be in a statistical mixture state. The smaller the purity is, the less pure the state will be. Further, when $T_{f}=0.5$ the field and the atom will be in a maximally entangled state. As we mentioned above $T_{f}$ gives information about entanglement of the components of the system, which forms the basis of experiments in the realm quantum information. Further, the disentanglement of the two quantum systems suggests interesting applications, e.g., in atomic states preparation through interacting quantum systems or in the choice of optimum time of flight in a micromaser to determine the cavity field [39]. Moreover, from the point of view of the quantum theory of measurement, the decoupled field state can be regarded as a pointeer basis since all the information about the atomic state has been transferred to the field [34]. We proceed, in Figs. 7 we plot the evolution of the $T_{f}$ for the given values of the interaction parameters. Comparison between the curves associated with $n=1$ and $n=0$ in Fig. 7a (for fixed value of $k$ ) shows that including Fock state $|1\rangle$ in the coherent optical cavity give rises to the oscillatory behaviour in $T_{f}$, changes the parity and achieve maximal entanglement at $T=2 \pi \sqrt{\langle\hat{n}(0)\rangle}$ (revival time). Actually, the oscillatory behaviour in $T_{f}$ occurs in a good correspondence with the revival in $\left\langle\hat{\sigma}_{z}(T)\right\rangle$. Further, for both cases $(n=0,1)$ the field collapses to a pure state at the middle of the collapse time $(\pi \sqrt{\langle\hat{n}(0)\rangle})$. Also the figure does not show a well-defined ringing revival for the case $n=1$, which is in contrast with the behaviour of the $\left\langle\hat{\sigma}_{z}(T)\right\rangle$. Furthermore, we have noted that increasing the value of $n$ will not give significant change in the overall behaviour of $T_{f}$ except 
increasing the oscillations. However, for the superimposed optical cavity, we have noted also that the state of the field never gets as pure as in the Fig. 7a and the system remains in a maximally (or partially) entangled state most of the time. On the other hand, Fig. $7 \mathrm{~b}$ is given for fixed value of $n$ and different values of $k$ as indicated. For the case $k=2, T_{f}$ evolves smoothly and the field collapses to an approximate pure state at $T=\pi / 4$ and $3 \pi / 4$, which is long lived as compared to those occurring at times multiples of $\pi$. As we mentioned above for $T=s \pi, s=0,1,2 . .$, the optical cavity field returns to its initial form. Furthermore, the field and the atom are long lived maximally entangled at $T=\pi / 2$ and $3 \pi / 2$. It is evident that $T_{f}$ has a systematic behaviour as $\left\langle\hat{\sigma}_{z}(T)\right\rangle$. Now we turn our attention to the case $k=4$. One can observe that $T_{f}$ displays pure states instantaneously with period $\pi / 2$, however, at $T=\pi / 4$ and $3 \pi / 4$, the field will be in a partially mixed state. Apart from these values of interaction time the field and the atom are approximately maximally entangled. The similarity and dissimilarity between the $T_{f}$ for the cases $k=2$ and $k=4$ are remarkable. On the other hand, for the case $k=3$, the field goes to approximate mixture states just after the interaction is turned on meaning that the field and the atom almost retain a strong entanglement all over interaction times.

In conclusion, the behaviour of $P(m, T)$ and $T_{f}$ are in a good agreement with each others. Also, the analysis of the purity gives information that could not be predicted by looking at the inversion or the photon-number distribution solely. We have not considered the influence of squeezing parameter in the present section since it generally increases the oscillatory behaviour in the $P(m, T)$.

\section{SUB-POISSONIAN STATISTICS AND QUADRATURE SQUEEZING}

In this section we discuss the sub-Poissonian statistics and quadrature squeezing for the system under consideration. Both of these quantities can be used as a measure for the nonclassical effects. Firstly, the sub-Poissonian statistics can be analyzed using Mandel $Q$ parameter [40] having the form

$$
Q(T)=\frac{\left\langle(\triangle \hat{n}(T))^{2}\right\rangle-\langle\hat{n}(T)\rangle}{\langle\hat{n}(T)\rangle} .
$$

Formula (28) characterizes the deviation from Poissonian statistics. It holds that $-1 \leq Q(T)<0$ for sub-Poissonian statistics (nonclassical effect), $Q(T)>0$ for super-Poissonian statistics (classical effect) and $Q(T)=0$ for Poissonian statistics (the standard one). Sub-Poissonian light can be measured by means of a set of photodetectors. Further, this light has several applications, e.g. in the gravitational wave detector and quantum nondemolition measurement, and can be generated 

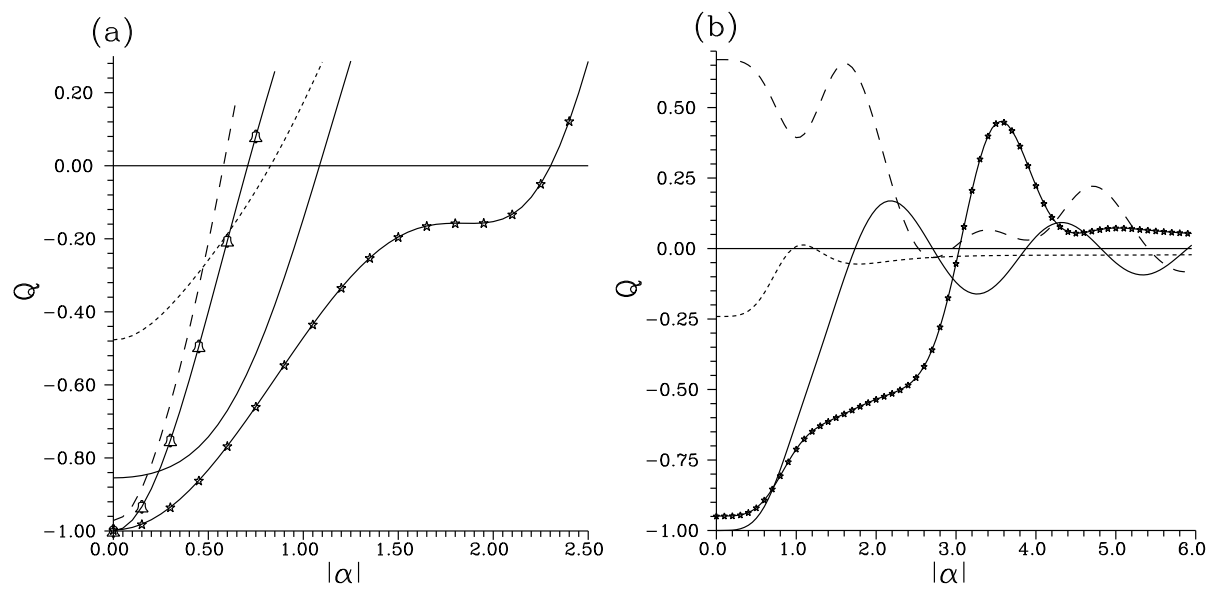

FIG. 8: Mandel $Q$ parameter against $|\alpha|$ when the optical cavity field is initially prepared in $\hat{D}(\alpha)|1\rangle$ (a) and even coherent state (b) for $T=1.578$ and for $k=1,2,3,4$ corresponding to solid, short-dashed, long-dashed and star-centered curves. In (a) the bell-centered curve is for zero-interaction time. Straight line is the sub-Poissonian bound.

in semiconductor lasers [41] and in the microwave region using masers operating in the microscopic regime [42].

Secondly, in order to investigate squeezing we define two quadrature operators as $\hat{X}=$ $\frac{1}{2}\left[\hat{a}+\hat{a}^{\dagger}\right], \quad \hat{Y}=\frac{1}{2 i}\left[\hat{a}-\hat{a}^{\dagger}\right]$. These quadratures relate to the conjugate electric and magnetic field operators of the electromagnetic wave and satisfy the commutation rule $[\hat{X}, \hat{Y}]=\frac{i}{2}$. Therefore, the uncertainty relation reads $\left\langle(\triangle \hat{X}(T))^{2}\right\rangle\left\langle(\triangle \hat{Y}(T))^{2}\right\rangle \geq \frac{1}{16}$. So we can say that the system is able to produce squeezing if the squeezing factor $F(T)=4\left\langle(\triangle \hat{X}(T))^{2}\right\rangle-1<0$ or $S(T)=4\left\langle(\triangle \hat{Y}(T))^{2}\right\rangle-1<0$. As is well known squeezed light can be measured by a homodyne detection in which the signal is superimposed on a strong coherent beam of the local oscillator. Furthermore, quite recently it has been shown experimentally that there is an evidence of squeezed light in the biological systems [43].

The required expectation values to treat these quantities can be calculated using the following relation

$$
\left\langle\hat{a}^{\dagger s}(T) \hat{a}^{s^{\prime}}(T)\right\rangle=\operatorname{Tr}\left[\hat{\rho}(T) \hat{a}^{\dagger s}(0) \hat{a}^{s^{\prime}}(0)\right],
$$

where $\hat{\rho}(T)$ is given by (13) and $s, s^{\prime}$ are positive integers. Before discussing the sub-Poissonian statistics for the present system it is worth reminding that for the standard JCM the sub-Poissonian statistics have been realized [10]. Furthermore, the establishment of such statistics for input squeezed coherent states relies on the competition between the squeezing parameter $r$ and coherent amplitudes $|\alpha|[5]$. In other words, as the values of $r$ increase the amount of sub-Poissonian 
statistics (resulting from the coherent component) is decreased and eventually disappeared. For the system under consideration the squeezing parameter plays the same role, i.e. it destroys the sub-Poissonian statistics. In general, we found that $Q(T)$ exhibits collapse-revival pattern as well as ringing revivals, which reflects the time evolution of the atomic inversion. In this case the photon statistics already oscillates between sub-Poissonian and super-Poissonian statistics based on the values of the interaction parameters. Here we would like to address two facts: (i) The system can enhance the sub-Poissonian interval for the initial sub-Poissonian states. (ii) The system can generate sub-Poissonian light for an initial non-sub-Poissonian superimposed state (e.g. even coherent states). Information about these facts is included in Figs. 8a and b for given values of the parameters. In these figures we have plotted Mandel $Q$ parameter in the stationary regime at specific value of interaction time, since the time-dependent coefficients in the present system are periodic in time. The selected value of the interaction time gives maximum sub-Poissonian statistics for the case $k=1$ when the optical cavity field is initially prepared in the even coherent states. From Fig. 8a, one can observe that the curves are smooth and the most sub-Poissonian statistics occur for $k=3$ and 4. Also this figure gives the coherent amplitude interval over which the sub-Poissonian light can be obtained for different values of the absorption parameter. It is evident that the intervals for the cases $k=1,2,4$ are greater than those for the initial one (compare different curves with the bell-centered one). This shows that how one can enhance the sub-Poissonian interval based on the value of the absorption parameter. Furthermore, the amount of sub-Poissonian statistics decreases almost exponentially as the magnitude $|\alpha|$ increases similar to that of the standard JCM [44]. Figure (b) carries information about the behaviour of the Mandel $Q$ parameter when the optical cavity field is initially in even coherent states. It is worth reminding that this state exhibits always super-Poissonian statistics. However, here through the evolution we can observe that the sub-Poissonian statistics are achieved giving their maximum values for $k=1,4$. In Fig. $8 \mathrm{~b}$ the curves include smooth oscillations resulting from the superposition principle. Further, the subPoissonian intervals are increased compared to those in the Fig. 8a, in particular, for $k=1,2,4$. The two-photon absorption case (short-dashed curve) almost exhibits steady-state sub-Poissonian statistics for large values of $|\alpha|$, whereas the case $k=3$ exhibits sub-Poissonian statistics only for the large values of $|\alpha|$. Finally, figures 8 show that there is no clear relation between the obtained sub-Poissonian statistics and the values of the absorption parameter $k$.

Now we turn our attention to discuss quadrature squeezing. Actually, it has been shown that squeezed light can be obtained in the standard JCM [9]. However, for input squeezed light with the atom initially prepared in excited atomic state the interaction destroys the initial squeezing, which 

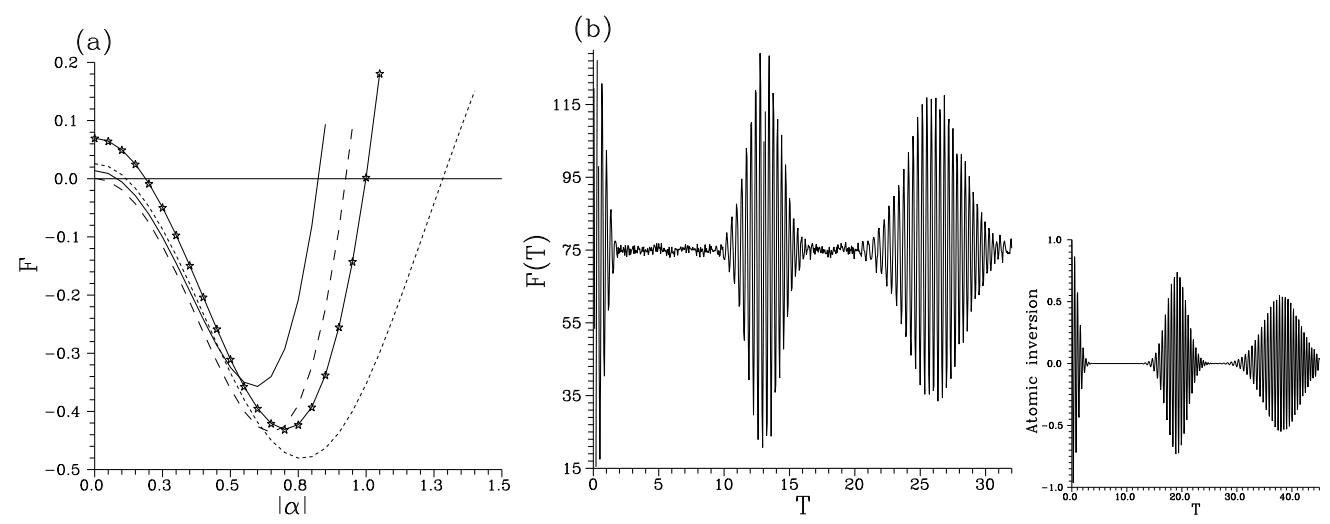

FIG. 9: The squeezing factor $F$ against $|\alpha|$ (a) and scaled time $T$ (b) for even coherent states. In (a) $(k, T)=(1,12.65)$ (solid curve), $(2,4.5)$ (short-dashed curve) $(3,7.7)$ (long-dashed curve), $(4,9.6)$ (starcentered curve) and the straight line is the squeezing bound. In (b) $(k, \alpha)=(3,6)$ and the inset is the atomic inversion of the case $(k, \alpha)=(1,6)$.

is inherent in the radiation field [19]. Here we note that squeezing can be obtained provided that the initial superimposed optical cavity is squeezed. Moreover, the system can switch squeezing from one quadrature to the other. The origin of this is in the periodic nature of the system. For example, even coherent state with real $\alpha$ exhibits squeezing in $Y$-quadrature provided that $0 \leq|\alpha|<2[45]$. In the present system squeezing can be transferred to $X$-quadrature independent of the values of $k$ (see Fig. 9a for given values of the parameters). In this figure the values of the interaction time have been chosen numerically in order to maximize the amount of squeezing in $F$. We proceed, from Fig. 9a for each value of $k, F$ starts from almost minimum-uncertainty state around $|\alpha|=0$ to increase the amount of squeezing until it exhibits its maximum squeezing, then starts to decrease the squeezing and eventually exhibits classical effect (i.e. squeezing completely disappears). Also from this figure the maximum (minimum) amount of squeezing occurs for the case $k=2$ for these chosen values of the parameters. Fig. 9b includes the dynamical behaviour for the $X$ quadrature for the case $(k,|\alpha|)=(3,6)$. It is obvious that there is no nonclassical negative values (, i.e. there is no squeezing). Nevertheless, $F(T)$ exhibits collapses and revivals, which (apart from the different scales) are very similar to those of the $\left\langle\hat{\sigma}_{z}(T)\right\rangle$ for the case $k=1$ (compare the behaviour of the inset in Fig. 9b with that of $F(T)$ ). To be more specific, qualitatively both these curves are equivalent in such a way that they collapse, remain quiescent, revive, collapse again and repeatedly undergo a complicated pattern of collapses and revivals for large interaction time, which is not included in the figure. Such type of equivalence leads to an interesting result: the collapse-revival phenomenon in the $\left\langle\hat{\sigma}_{z}(T)\right\rangle$ for the standard JCM can be detected indirectly 
via measuring the quadrature squeezing for three-photon absorption case through the homodyne detection. It is worth mentioning that observation of collapses and revivals were performed using the one-atom mazer [2], which is more sophisticated than the dynamics of the JCM.

\section{WIGNER FUNCTION}

In this section we investigate the behaviour of the Wigner $(W)$ function for the system under consideration. Actually, the $W$ function is informative, sensitive to the interference in phase space, and can give a prediction to the possible occurrence of the nonclassical effects. Furthermore, this function can be constructed experimentally using the optical homodyne tomography [46].

The $W$ function is defined [47] as

$$
W(x, p, T)=\frac{1}{2 \pi} \int_{-\infty}^{\infty} d \zeta \exp (-i p \zeta)\left\langle x+\frac{\zeta}{2}\left|\hat{\rho}_{f}(T)\right| x-\frac{\zeta}{2}\right\rangle
$$

where we have assumed that $\hbar=\omega_{0}=1$. On substituting the wavefunction obtained from (13) into (30) and using both the wavefunction of the Fock state as

$$
\langle x \mid n\rangle=\frac{\exp \left(-\frac{x^{2}}{2}\right)}{\sqrt{\pi^{\frac{1}{2}} 2^{n} n !}} \mathrm{H}_{n}(x)
$$

and the identity [48]

$$
\int_{-\infty}^{\infty} \exp \left(-\zeta^{2}\right) \mathrm{H}_{m}\left(\zeta+z_{1}\right) \mathrm{H}_{n}\left(\zeta+z_{2}\right) d \zeta=2^{n} \sqrt{\pi} m ! z_{2}^{n-m} \mathrm{~L}_{m}^{n-m}\left(-2 z_{1} z_{2}\right), \quad m \leq n
$$

we arrive at

$$
\begin{aligned}
& W(x, p, T)=\frac{\exp \left(-|\chi|^{2}\right)}{\pi}\left\{\sum_{m, m^{\prime}=0}^{\infty}(-1)^{m^{\prime}} \chi^{m-m^{\prime}} 2^{\frac{m-m^{\prime}}{2}} C_{m}(\alpha, r, n, \epsilon) C_{m^{\prime}}^{*}(\alpha, r, n, \epsilon)\right. \\
& {\left[\cos [T \sqrt{h(m, k)}] \cos \left[T \sqrt{h\left(m^{\prime}, k\right)}\right] \sqrt{\frac{m^{\prime} !}{m !}} \mathrm{L}_{m^{\prime}}^{m-m^{\prime}}\left(2|\chi|^{2}\right)\right.} \\
& \left.\left.+(-1)^{k} \sin [T \sqrt{h(m, k)}] \sin \left[T \sqrt{h\left(m^{\prime}, k\right)}\right] \sqrt{\frac{\left(m^{\prime}+k\right) !}{(m+k) !}} \mathrm{L}_{m^{\prime}+k}^{m-m^{\prime}}\left(2|\chi|^{2}\right)\right]\right\},
\end{aligned}
$$

where $\chi=x+i p$.

Before discussing the behaviour of (33) it is worth mentioning that the $Q$ distribution function of the standard JCM with and without cavity damping have been investigated in [49, 50, 51, 52]. It has been shown, for the non-damping case, that the initial shifted Gaussian distribution splits into two distributions, which counter-rotate on a circle in the complex plane of the distribution. At the opposite end of the circle the two peaks collide, they split again, and so forth. This behaviour 
is closely connected to the revival-collapse phenomenon in the $\left\langle\hat{\sigma}_{z}(T)\right\rangle$, where collapses of the Rabi oscillations occur during splitting of the distribution and the revivals occur during collision of the peaks of the distribution. As the system evolves the width of the peaks become broader. When they spread over the whole circle no collision occurs anymore and distinct collapses and revivals are no longer observed. Also splitting in the $Q$ distribution for the JCM is explained based on the interpretations of the density matrix [53]. Further, it is worth mentioning that the generation of superposition states in the standard JCM has been investigated using this function. For the damping case the counter-rotating peaks spiral into the origin till finally the stationary distribution is reached. Employing $W$ function for JCM has stimulated few efforts, in particular, for the damping case [49]. Here we provide a connection between the evolution of both $\left\langle\hat{\sigma}_{z}(T)\right\rangle$ and the value of the $W$ function at the origin for the two following cases.

Case (i): when the absorption parameter is odd number, i.e. $k=1,3,5, \ldots$ From (33) we can write the following expression

$$
W(0, T)=\frac{1}{\pi} \sum_{m=0}^{\infty}(-1)^{m} P(m) \cos [2 T \sqrt{h(m, k)}],
$$

where only the diagonal terms survive. Careful examination to (34) leads to the following facts: for the pair-photon states (i.e. even or odd states) (34) can be rewritten as

$$
W_{ \pm}(0, T)= \pm \frac{1}{\pi}\left\langle\hat{\sigma}_{z}(T)\right\rangle
$$

where the positive (negative) sign refers to even (odd) case. This expression indicates that when $W(0, T) \simeq 0 \quad(\neq 0)$ the collapse (revival) occurs in $\left\langle\hat{\sigma}_{z}(T)\right\rangle$. It is obvious that when $W(0, T)= \pm 1 / \pi$ the amplitude of $\left\langle\hat{\sigma}_{z}(T)\right\rangle$ is maximum. Furthermore, the value of $W$ function at the origin exhibits nonclassical negative values in the course of revival times, which means that the nonclassical effects most probable occur during this time. On the other hand, for the non-pair-photon states the opposite behaviour occurs. For instance, for the standard JCM $W(0, T) \neq 0 \quad(\simeq 0)$ the collapse (revival) occurs in $\left\langle\hat{\sigma}_{z}(T)\right\rangle$. These ensure that Schrödinger-cat state can be generated during the collapse time, where the establishment of the interference in phase space is quite obvious. This fact will be made clear shortly.

Case (ii): when the absorption parameter is even number, i.e. $k=2,4, \ldots$. In this case the origin of the $W$ function is localized in phase space (time independent) having its initial value $W(0, T)=W(0,0)$. This leads to a novel result, which is: the nonclassical states whose $W$ functions 

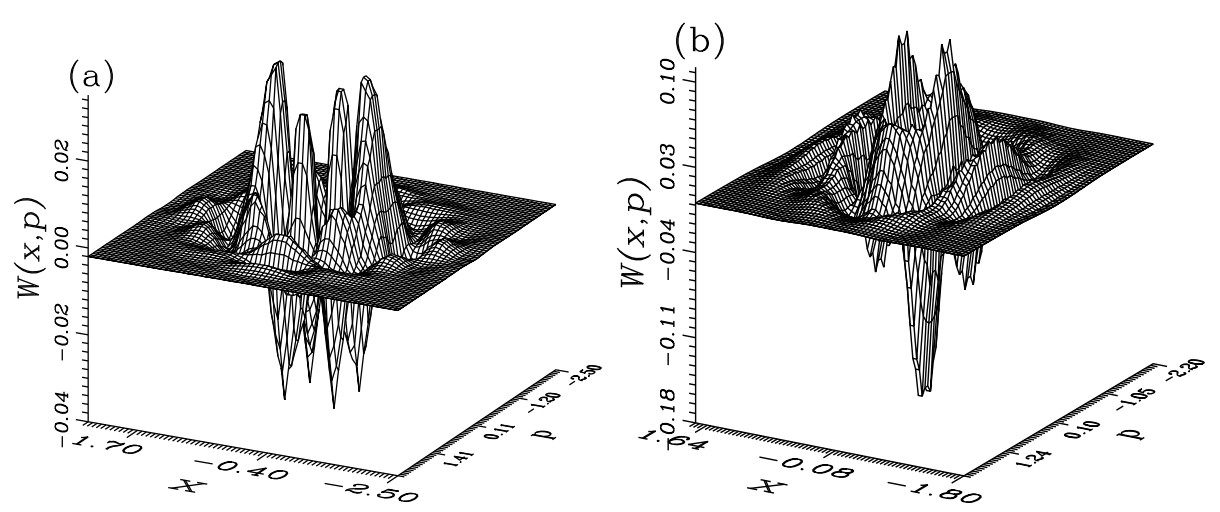

FIG. 10: $W$ function of the even displaced Fock state for $\alpha=3, k=1, n=1$ and for: a) $T=4.599998$ (collapse time) and b) $T=12.60001$ (revival time).

have negative values at the origins (e.g. odd coherent state) will be always nonclassical when they are evolving through the JCM with even absorption parameter. Of course the chaotic behaviour (and/or overlap of the successive revivals) in $\left\langle\hat{\sigma}_{z}(T)\right\rangle$ can be recognized in the evolution of the $W$ function at the origin when $\left|W\left(0, T_{1}\right)\right| \simeq\left|W\left(0, T_{2}\right)\right|$ such that $T_{1} \geq T_{2}$. It is worth referring to the nonlinear version scheme of a single-atom homodyne detector in which the ionization probability of a single test atom is given in terms of the Wigner characteristic function of the field [54]. In Figs. 10 a and b we plot $W$ function for even displaced number state for $T=4.599998$ (collapse time) and 12.60001 (revival time), respectively, for the given values of the parameter. The values of the interaction time are chosen numerically. Actually, $W$ function of one-photon even coherent state consists of two inverted holes, with more pronounced negative values, and interference fringes inbetween. In Figs. 10 according to the evolution of the dynamical system such shape is destroyed and the $W$ function becomes more structuralized, which is more pronounced for the collapsed case Fig. 10a. To be more specific, Fig. 10 a includes multi-peak structure ensuring the generation of the Schrödinger-cat states. Also for this case we find that $W(0, T) \simeq 0$. Conversely, Fig. $10 \mathrm{~b}$ includes more pronounced negative values at the origin of phase space. These facts are in a good agreement with the above conclusions.

The second issue we would like to address here is that it has been shown that cat states can be generated at one-half of the revival time in the standard JCM [34, 35, 36, 37]. One of the ways is the investigation of the $Q$ function. Nevertheless, this function does not include a complete information about the system as the $W$ function includes. Now we give the form of the $W$ function for such type of cat states. We do so for the standard JCM, i.e. $k=1$ and $\epsilon=n=r=0$, with strong intensity in such a way that the harmonic approximation is applicable. To deal with the 
second part of (33) some tricks should be performed, e.g. replacing $C_{m}$ by $C_{m+1}$ since we are working in the strong-intensity regime such a transformation is relevant. On using (15) in addition to the properties of the Laguerre polynomial (33) reduces to

$$
\begin{aligned}
& W(x, p, T)=\frac{1}{2 \pi} \exp \left[-\left(x-\varepsilon_{1}\right)^{2}\right]\left\{\exp \left[-\left(p-\varepsilon_{2}\right)^{2}\right]+\exp \left[-\left(p+\varepsilon_{2}\right)^{2}\right]\right. \\
& \left.-2 \exp \left(-p^{2}\right) \sin \left(\frac{T \eta_{2}}{2}\right) \sin \left[T\left(\eta_{1}-\frac{\eta_{2}}{2}\right)+2 \alpha\left(\sqrt{2} x-\alpha \cos \left(\frac{T \eta_{2}}{2}\right)\right) \sin \left(\frac{T \eta_{2}}{2}\right)\right]\right\},
\end{aligned}
$$

where

$$
\varepsilon_{1}=\sqrt{2} \alpha \cos \left(\frac{T \eta_{2}}{2}\right), \quad \varepsilon_{2}=\sqrt{2} \alpha \sin \left(\frac{T \eta_{2}}{2}\right)
$$

We should stress that for the best of our knoweldge this is the first time for which the exact form of the $W$ function, i.e. (36), of the generated cat state in the microcavity is given. Expression (36) shows that the peaks centers move on the circle controlled by (37). In fact, the appearance of the factor $\sqrt{2}$ in the definition of the circle is related to the definition of $W$ function (30), however, if one uses the transformation $(x, p) \rightarrow \sqrt{2}(q, y)$ then the relations (36) and (37) would be consistent with those obtained earlier for the $Q$ function, e.g. [52], provided that the prefactor $1 /(2 \pi)$ is replaced by $1 / \pi$. Such a prefactor is connected with the transformation in the integration (30). Expression (36) shows that at the revival times (i.e. $T=2 \pi / \eta_{2}$ ) the two peaks collide, whereas at one-half revival time the well-known shape of the cat-state $W$ function is obtained, which is two Gaussian bells corresponding to statistical mixture of individual composite states and interference fringes inbetween arising from the contribution of the overlapping between different components of the state. It is worth referring to [52], in which an analytical closed form for the $Q$ function is derived by applying the method of steepest decent and saddle-point integration in the limit of high photon numbers. The final remark, the asymptotic form for the $W$ function related to the two-photon absorption case can be obtained from (36) by simply setting $\eta_{1}=3$ and $\eta_{2}=2$. In this case the $W$ function is intensity independent and its behaviour is systematic as that of $\left\langle\hat{\sigma}_{z}(T)\right\rangle$.

\section{CONCLUSIONS}

In this paper we have discussed the quantum properties for superposition of squeezed displaced number states defined by (11) against the lossless multiphoton Jaynes-Cummings model. We have assumed that the atom is initially prepared in the excited state. In addition to the characteristic features of the system the investigation gives generalization to various results in the literatures. 
The investigated quantities are the atomic inversion, photon-number distribution, purity, Mandel $Q$ parameter, quadrature squeezing and $W$ function. The main conclusions are the followings: Atomic inversion exhibits revival-collapse pattern as well as echoes, which are readout of the photon-number distribution. Furthermore, including squeezing in the superimposed optical cavity can cause revivals and collapses in the atomic inversion. The photon-number distribution shows that various forms of cat states can be generated via the system. Also the entanglement and disentaglement between the field and atom occur periodically since the system is characterized by the periodic exchange of energy between the atom and the cavity mode. Such behaviour has been confirmed in the purity evolution. Actually, the behaviour of these quantities basically depends upon the values of the absorption parameter $k$, which results in the functional dependence of the Rabi frequency on the mean photon number. The sub-Poissonian statistics occur regardless of the values of $k$ provided that $\alpha$ is finite. Also squeezing in the quadrature squeezing can be occurred and switched between the two quadratures provided that the initial superimposed optical cavity is squeezed. Furthermore, the evolution of the quadrature squeezing for the case $k=3$ exhibits revivals and collapses similar to those of the atomic inversion of the standard JCM. Actually, this is a novel result and its consequence is that the revival-collapse phenomenon exhibited for the atomic inversion can be measured by homodyne detection via three-photon microcavity [55]. With respect to $W$ function we have found some novel results, which can be summarized as follows: There is a direct relation between the evolution of the values of the $W$ function at the origin in phase space and the evolution of $\left\langle\hat{\sigma}_{z}(T)\right\rangle$ when the absorption parameter $k$ is odd number. This suggests that the atomic inversion can be detected via both the homodyne tomography [46] and trapped ion [56]. It is worthwhile mentioning that in the trapped ion methods a proposal for measuring the $W$ function at the origin of the phase space for a single photon field is given [56] and this would be relevant for the task. On the other hand, for even absorption parameter the origin of the $W$ function in phase space is localized and thus the nonclassical states whose $W$ functions include negative values at the origin will be always nonclassical when they are evolving with the JCM. Furthermore, for the first time, as far as we know, we have given the explicit form of the $W$ function of the generated cat state in the microcavity. 


\section{References}

[1] Jaynes E T and Cummings F W 1963 Proc. IEEE 5189.

[2] Rempe G, Walther H and Klein N 1987 Phys. Rev. Lett. 57353.

[3] Buck B and Sukumar C V 1981 Phys. Lett. A 81132.

[4] Gerry C C and Moyer P J 1988 Phys. Rev. A 385665.

[5] Mahran M H and Obada A-S 1989 Phys. Rev. A 40 2215; Maqbool T and Razmi M S K 1991 Phys. Rev. A 44 6147; Obada A-S and Abdel-Hafez A M 1991 Phys. Rev. A 446017.

[6] Obada A-S and Abdel-Hafez A M 1986 Physica A 139 593; Knight P L 1986 Phys. Scr. 33 51; Phoenix S J D and Knight P L 1990 J. Opt. Soc. Am. B 7 116; Bužek V and Jex I 1989 Quant. Opt. 2 147; Benivegna G B, Messina A and Napoli A 1994 Phys. Lett. A 194353.

[7] Quang T, Knight P L and Bužek V 1991 Phys. Rev. A 44 6092; Barnett S M and Knight P L 1986 Phys. Rev. A 332444.

[8] Eberly J H, Narozhny N B and Sanchez-Mondragon J J 1980 Phys. Rev. Lett. 44 1323; Narozhny N B, Sanchez-Mondragon J J and Eberly J H 1981 Phys. Rev. A 23 236; Yoo H I, Sanchez-Mondragon J J and Eberly J H 1981 J. Phys. A 14 1383; Yoo H I and Eberly J H 1981 Phys. Rep. 118239.

[9] Meystre P and Zubairy M S 1982 Phys. Lett. A 89A 390.

[10] Kim M S 1993 J. Mod. Opt. 401331.

[11] Garraway B M, Sherman B, Moya-Cessa H, Knight P L and Kurizki G 1994 Phys. Rev. A 49 535; Kozhekin A, Kurizki G and Sherman B 1996 Phys. Rev. A 543535.

[12] Shore B W and Knight P L 1993 J. Mod. Opt. 401195.

[13] Vidiella-Barranco A, Moya-Cessa H and Bužek V 1992 J. Mod. Opt. 391441.

[14] Gerry C C and Hach III E E 1993 Phys. Lett. A 1791.

[15] Joshi A and Singh M 1995 J. Mod. Opt. 42775.

[16] Kim M S, De Oliveira F A M and Knight P L 1990 J. Mod. Opt. 37659.

[17] Milburn G J 1984 Opt. Act. 31671.

[18] Satyanarayana M V, Rice P, Vyas R and Carmichael H J 1989 J. Opt. Soc. Am. B 6228.

[19] Cohen D, Ben-Aryeh Y and Mann A 1994 Phys. Rev. A 492040.

[20] Obada A-S F, Abd Al-Kader G M 1999 J. Mod. Opt. 46 263; Gou S-C, Knight P L 1996 Phy. Rev. A 54 1685; Gou S-C, Steinbach J, Knight P L 1996 Phy. Rev. A 54 4315, ibid R1014, ibid 1997553719.

[21] El-Orany F A A 1999 Czech. J. Phys. 491145.

[22] El-Orany F A A, Peřina J and Abdalla M S 200 Quant. Semiclas. Opt. 2545.

[23] El-Orany F A A, Peřina J and Abdalla M S 1999 J. Mod. Opt. 461621.

[24] Kaicheng Z, Huiqin T, Cuiliang L, Duzhi H, Xinguag L, Xiangru L 1996 J. Mod. Opt. 43323.

[25] D'Ariano G, Rasetti M and Vadacchino M 1985 Phys. Rev. D 32 1034; Katriel J, Solomon A I, D'Ariano 
G and Rasetti M 1986 Phys. Rev. D 34 2332; D'Ariano G and Rasetti M 1987 Phys. Rev. D 35 1239; Katriel J, Rasetti M and Solomon A I 1987 Phys. Rev. D 351248.

[26] Katriel J, Rasetti M and Solomon A I 1987 Phys. Rev. D 352601.

[27] Dodonov V V 2002 Quant. Semiclass. Opt. 4 R1.

[28] Scully M O and Zubairy M S: "Quantum Optics" (Cambridge University Press: 1997).

[29] Peřina Jr and Peřina J: "Quantum statistics of nonlinear optical couplers", Progress in Optics, Vol. 41, ed. E. Wolf, (Amsterdam: Elsevier 2000).

[30] Moya-Cessa H 1995 J. Mod. Opt. 42 1741; Obada A-S F and Abd Al-Kader G M 1998 Acta Phys. Slov. 48583.

[31] Zheng S-B and Guo G-C 1996 Quantum Semiclass. Opt. 8951.

[32] Fleischhauer M and Schleich W P 1993 Phys. Rev. A 474258.

[33] Joshi A and Lawande S V 1993 Phys. Rev. A 48 2276, and references therein.

[34] Gea-Banacloche J 1991 Phys. Rev. A 445913.

[35] Bužek V, Moya-Cessa H and Knight P L 1992 Phys. Rev. A 458190.

[36] Bužek V and Hladký 1993 J. Mod. Opt. 401309.

[37] Zaheer K and Wahiddin M R B 1994 J. Mod. Opt. 41151.

[38] Schleich W, Pernigo M and Kien F L 1991 Phys. Rev. A 442172.

[39] Phoenix S J D and Knight P L 1991 Phys. Rev. A 446023.

[40] Mandel L 1979 Opt. Lett. 4205.

[41] Yamamoto Y and Machida S 1987 Phys. Rev. A 355114.

[42] Rempe G, Schmidt-Kaler F and Walther H 1990 Phys. Rev. Lett. 642783.

[43] Popp F A, Chang J J, Herzog A, Yan Z and Yan Y 2002 Phys. Lett. A 29398.

[44] Hillery M 1987 Phys. Rev. A 354186.

[45] Hach III E E and Gerry C C 1993 J. Mod. Opt. 402351.

[46] Leonhardt U: "Measuring the Quantum State of Light" (Cambridge: University Press 1997).

[47] Schleich W P 2001 : " Quantum Optics in Phase Space" (Berlin: WILEY-VCH 2001).

[48] Gradshteyn I S G and Ryzhik I M: " Table of Integrals, Series, and Products" (Boston: Academic Press 1994).

[49] Eiselt J and Risken H 1989 Opt. Commun. 72351.

[50] Eiselt J and Risken H 1991 Phys. Rev. A 43346.

[51] Eiselt J and Risken H 1991 Phys. Rev. A 44 4623; Werner M J and Risken H 1991 Quant. Opt. 3185.

[52] Miller C A, Hilsenbeck J and Risken H 1992 Phys. Rev. A 464323.

[53] Matsuo K 1994 Phys. Rev. A 50649.

[54] Wilkens M and Meystre P 1991 Phys. Rev. A 433832.

[55] El-Orany F A A 2002 Phys. Rev. A (submitted).

[56] Lutterbach L G and Davidovich L 1997 Phys. Rev. lett. 78 2547; Nogues G, Rauschenbeutel A, Osnaghi S, Bertet P, Brune M, Raimond J M, Haroche S, Lutterbach L G and Davidovich L 2000 Phys. Rev. A 
62054101. 